\title{
Toward a Perfect Single-Photon Source
}

\section{Semiconductor quantum dot emits photons that are squeezed below the fundamental noise limit.}

\section{By Rachel Berkowitz}

( ptical-based quantum technologies use individual photons to encode and transmit information. However, even the best existing tools for generating photons produce beams whose number of photons fluctuates. This fluctuation creates uncertainty in the timing and intensity of a received signal. Hui Wang and colleagues at the University of Science and Technology of China have now developed a device that suppresses those fluctuations, allowing it to produce a more precise number of photons [1]. The device could be used as a new standard for quantum computing and high-precision quantum measurements.

In the classical world, it was thought to be straightforward to generate a well-defined amount of radiation in a given time period. But at the quantum scale, inherent uncertainty in a photon's energy and phase reduces that precision and results in a blurred photon count. To solve this problem, Wang and his colleagues designed a photon source that could eliminate the blurring.

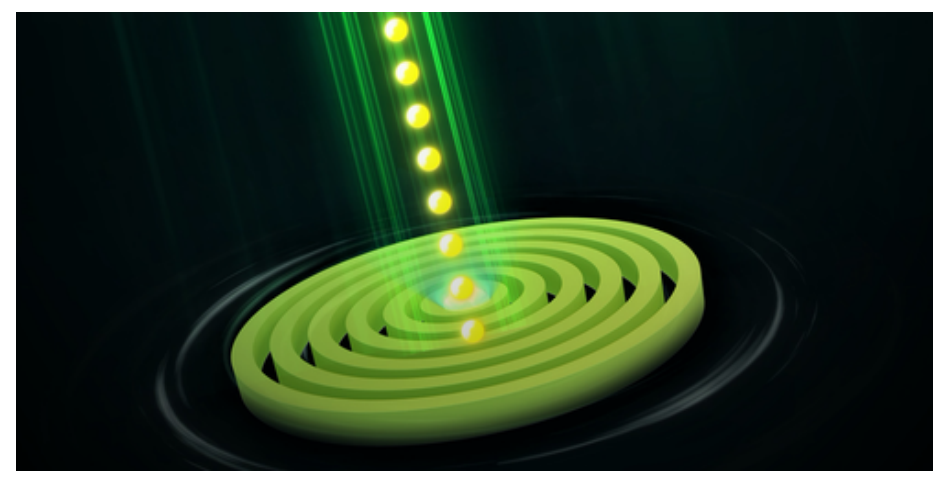

Credit: C.-Y. Lu/University of Science and Technology of China
The researchers coupled a semiconductor quantum dot to the center of a $2-\mu \mathrm{m}$-diameter cavity using tiny micropillars. When excited by a laser, the quantum dot emitted photons, which were tightly confined in the cavity. This confinement helped reduce photon loss when the photons were subsequently funneled into an optical fiber and measured with a semiconducting nanowire detector.

This so-called squeezing reduced the intensity of the fluctuations by about $13 \%$ compared with an unconfined source. This reduction translated into a more precise measurement of the average photon rate- 17.5 photons per 1.0 $\mu \mathrm{s}$, with a standard deviation of 3.65 photons. The team says that their "highly efficient, simple-to-produce" device could aid a large-scale international effort to reformulate the SI unit of light intensity in terms of photon number

Rachel Berkowitz is a Corresponding Editor for Physics based in Seattle, Washington, and Vancouver, Canada.

\section{REFERENCES}

1. H. Wang et al., "Observation of intensity squeezing in resonance fluorescence from a solid-state device," Phys. Rev. Lett. 125, 153601 (2020). 